\title{
Cost-effectiveness of mometasone furoate nasal spray in the treatment of acute rhinosinusitis
}

\section{*Johanna Svensson', Johan Lundberg' ${ }^{2}$ Petter Olsson³, Pär Stjärne”, Gunnel Ragnarson Tennvall ${ }^{1}$}

\author{
${ }^{1}$ IHE, The Swedish Institute for Health Economics, Lund, Sweden \\ ${ }^{2}$ MSD, Sollentuna, Sweden \\ ${ }^{3}$ Department of Clinical Science, Intervention and Technology (CLINTEC), Division of Otorhinolaryngology, Karolinska Institutet, \\ Stockholm, Sweden
}

Received 26th January 2012; revised 16th May 2012; accepted 12th July 2012; online 21st September 2012

\begin{abstract}
Background: Acute rhinosinusitis is a common disease with an increasing incidence rate. It causes substantial costs to the individual and to society through healthcare consumption and absence from work. The use of antibiotics is widespread in the treatment of acute rhinosinusitis, but increasing bacterial resistance is an argument for restricting excessive use of antibiotics.

Aims: The aim of this study was to analyse the cost-effectiveness of mometasone furoate nasal spray (MFNS) compared with amoxicillin or non-active treatment of mild to moderate acute rhinosinusitis in a Swedish setting.

Methods: A cost-effectiveness model was developed to capture the costs and health-related quality of life (HRQoL) over a 15-day period. Acute rhinosinusitis was modelled as changes in the Major Symptom Score. The model takes on a societal perspective in a Swedish setting.

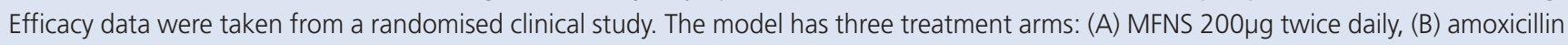
$500 \mathrm{mg}$ three times daily, and (C) placebo. Information about resource utilisation and HRQoL was taken from a recent observational study.

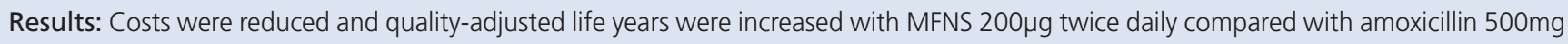
three times daily. MFNS was cost-saving or cost-effective compared with amoxicillin or non-active treatment in the sensitivity analyses regardless of the HRQOL measurement used.

Conclusions: This study shows that treatment with MFNS $200 \mu \mathrm{g}$ twice daily results in lower costs and improved HRQoL in acute rhinosinusitis compared with amoxicillin or self-medication.

(C) 2012 Primary Care Respiratory Society UK. All rights reserved.

J Svensson et al. Prim Care Respir J 2012; 21(4): 412-418

http://dx.doi.org/10.4104/pcrj.2012.00085
\end{abstract}

Keywords acute rhinosinusitis, cost-effectiveness, treatment pattern

\section{Introduction}

Acute rhinosinusitis is an inflammation of the mucosa of the nose and paranasal sinuses of viral or bacterial origin. Rhinosinusitis is defined as acute when symptoms escalate after 5 days and/or remain after 10 days, but not more than 12 weeks. ${ }^{1}$ It is a common disease with an increasing incidence rate that may be connected to the increasing frequency of allergic rhinitis. ${ }^{1}$ Although acute rhinosinusitis often is a self-healing disease, it causes substantial costs to the individual and to society through healthcare consumption and absence from work. ${ }^{2}$ The predominant symptoms and signs of acute rhinosinusitis are nasal congestion and nasal discharge, respectively. ${ }^{1}$
It is difficult to distinguish between viral and bacterial infection without puncture of the sinus, so there is usually an element of uncertainty in the diagnosis of bacterial acute rhinosinusitis. Treatment options include nasal steroids and antibiotics, as well as conventional treatments such as nasal decongestants, nasal lavage, and analgesics aimed at treating symptoms. The use of antibiotics is widespread. A Swedish study shows that antibiotics were prescribed in more than $90 \%$ of cases of acute sinusitis, only declining marginally from $93.7 \%$ in 2000 to $91.6 \%$ in $2005 .{ }^{3}$ Increasing bacterial resistance is an argument for restricting excessive use of antibiotics. Other factors in favour of the use of anti-inflammatory

\footnotetext{
* Corresponding author: Ms Johanna Svensson, IHE, The Swedish Institute for Health Economics, P.O. Box 2127, SE-220 02 Lund, Sweden. Tel: +46 (0)4632 9100 Fax: +46 (0)46121604 E-mail: js@ihe.se
} 
nasal steroids in acute rhinosinusitis in comparison with antibiotics are the efficacy, ${ }^{14}$ safety profile, and the message to the patient that acute rhinosinusitis is mainly an inflammatory disease rather than infectious. The two most common adverse events with nasal steroids are headache and mild epistaxis. No increase in the risk of bacterial infections and recurrence or exacerbation of acute rhinosinusitis has been seen after mometasone furoate nasal spray (MFNS) treatment compared with amoxicillin. ${ }^{4}$ According to current European guidelines, the recommended treatment for mild acute rhinosinusitis is symptomatic, with nasal steroids advised in moderate cases.' Antibiotics should only be added if severe symptoms are present (e.g. fever $>38^{\circ} \mathrm{C}$ or severe pain). The evidence for the efficacy of decongestants in the treatment of acute rhinosinusitis in adults is poor. Since there is good evidence that acute rhinosinusitis can be successfully treated without antibiotics, the disease is now commonly seen as a target area where the consumption of antibiotics can be reduced. ${ }^{1,3,5}$ There are only a few cost-effectiveness studies of the treatment of acute rhinosinusitis ${ }^{6}$ and, to our knowledge, none on MFNS.

The aim of this study was to analyse the cost-effectiveness of MFNS compared with amoxicillin or other pharmacological and nonpharmacological treatment (self-medication or non-active treatment) in the treatment of mild to moderate acute rhinosinusitis in a Swedish setting.

\section{Methods}

\section{Health economic model}

A health economic model was developed to capture the costs and health-related quality of life (HRQoL) over a 15-day period. Acute rhinosinusitis was modelled as changes in the Major Symptom Score (MSS). ${ }^{4}$ MSS consists of five questions concerning rhinorrhoea, postnasal drip, nasal congestion, sinus headache, and facial pain. For each question there is a 4-level severity scale ranging from 0 to 3 (none, mild, moderate, severe). The MSS is the sum of the scores of the five questions (minimum 0, maximum 15). The model takes on a societal perspective in a Swedish setting.

The model was constructed as daily changes in MSS over a 15day period and starts when the diagnosis of acute rhinosinusitis is set and either treatment alternative is decided. The reason for not stretching the time frame from the onset of symptoms was that acute rhinosinusitis was defined as symptoms ongoing for at least 7 days. A cost-effectiveness analysis compares the costs and effects measured in quality-adjusted life years (QALYs) of one treatment with another. QALYs were obtained by dividing the HRQoL weight, measured by EQ-5D and EQ-VAS, corresponding to the mean MSS value by 365. QALYs and costs were calculated per day and MSS and summed by treatment arm over the 15-day period. The difference between the treatment arms in MSS thus generates a difference in costs and HRQoL. The MSS Utility Index (MSSUI) is a disease-specific HRQoL instrument. ${ }^{7}$ This was added to the model in the same way as EQ-5D and EQ-VAS.

\section{Input data}

Efficacy data were taken from a randomised clinical study. ${ }^{4}$ The

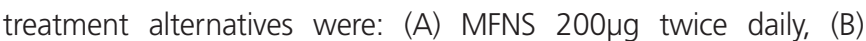

amoxicillin 500mg three times daily, and (C) placebo. MFNS/placebo was given for 15 days and amoxicillin for 10 days. After 15 days,

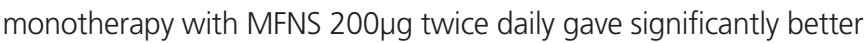
treatment effects than amoxicillin or placebo. The study further

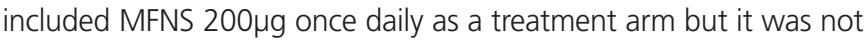
found to be superior to amoxicillin. ${ }^{4}$ In the model the placebo arm is referred to as non-active treatment since it is reasonable to assume that patients in real life who are not treated with intranasal steroids or amoxicillin still use prescription-free drugs such as analgesics and nasal decongestants.

Information about resource utilisation and HRQoL were taken from an observational, multicentre, prospective, non-randomised study in primary care aimed at describing symptoms, treatment patterns, HRQoL, and costs in patients with acute rhinosinusitis without signs and symptoms of severe bacterial infection. ${ }^{8}$ Data on disease severity, resource use, and HRQoL were collected at the first physician visit and at follow-up $15( \pm 2)$ days later. Baseline patient characteristics were similar to those reported for subjects in the clinical study (baseline mean MSS 8.34 and 8.39 and mean age 35 and 45 years in the clinical study and the observational study, respectively). ${ }^{4}$ In the model we based calculations on subjects with MSS at follow-up (i.e. 143 patients). The time frame was censored to a maximum of 22 days to follow-up. Intervention drug costs were calculated according to doses in the randomised clinical study. ${ }^{4}$ Amoxicillin treatment incurs the cost of two packs of 28 capsules (i.e. two capsules short of the prescribed dose). There is, however, a lack of evidence that antibiotic treatment of acute rhinosinusitis needs to go on for a full 10 days. ${ }^{9}$

Costs included direct costs (inpatient, outpatient, drugs and transportation) and indirect costs (productivity losses) (see Table 1 for unit prices). Concomitant medications in the observational study included decongestant tablets, decongestant sprays, allergy tablets/antihistamines, and analgesics. The cost of pharmaceuticals is based on the price of the smallest pack size, ${ }^{10}$ except for analgesics where it was assumed that patients would have a supply at home and thus only the daily cost was used to estimate cost. Indirect costs were calculated according to the human capital approach. The days of lost productivity were assigned the value of the mean daily income including social fees." All costs are presented in Swedish krona (SEK) 2010. The mean exchange rate in 2010 was 1 SEK = 0.105 Euros $=$ US $\$ 0.139$ (Riksbanken, the Swedish Central Bank).

\section{Health-related quality of life}

The EQ-5D weights were calculated using Dolan's tariff. ${ }^{12,13}$ The values on the EQ-VAS scale were divided by 100 to obtain HRQoL weights and facilitate a comparison with the EQ-5D weights. MSSUI was calculated using the tariff suggested by Revicki and colleagues.? EQ-5D was chosen as the base case utility measurement. HRQoL weights range between 0 and 1 , where 1 represents best imaginable health and 0 represents death. Negative values are possible with the EQ-5D tariff. They represent health states worse than death.

\section{Statistical analyses}

HRQoL and daily resource use were taken from a follow-up patient questionnaire. ${ }^{8}$ The statistical relationship between costs and MSS 
Table 1. Unit prices (SEK, 2010)

\begin{tabular}{|c|c|c|c|}
\hline & Unit price & Year & Reference \\
\hline Outpatient visits* & & & $22-24$ \\
\hline General practitioner & 1,472 & 2010 & \\
\hline ENT specialist & 1,524 & 2010 & \\
\hline ENT specialist, revisit & 1,008 & 2010 & \\
\hline Physician visit, ER & 2,679 & 2010 & \\
\hline Other healthcare professional & 694 & 2010 & \\
\hline Inpatient stay* & & & $22-24$ \\
\hline Day in hospital & 6,278 & 2010 & \\
\hline \multicolumn{4}{|l|}{ Transportation } \\
\hline Car, cost $/ \mathrm{km}$ & 3 & 2010 & \\
\hline Public transportation $<6 \mathrm{~km}^{\dagger}$ & 24 & 2010 & 25,26 \\
\hline Public transportation $>6 \mathrm{~km}^{\dagger}$ & 51 & 2010 & 25,26 \\
\hline \multicolumn{4}{|l|}{ Indirect costs } \\
\hline $\begin{array}{l}\text { Productivity losses including social } \\
\text { fees, per day }\end{array}$ & 1,992 & 2008 & 11 \\
\hline Pharmaceuticals, price per pack & & & 27 \\
\hline Antibiotics (generic) $^{\ddagger}$ & 120 & 2010 & \\
\hline Amoxicillin & 98.5 & 2010 & \\
\hline Nasal steroids/MFNS & 166 & 2010 & \\
\hline Decongestant tablet & 65 & 2010 & \\
\hline Decongestant spray ${ }^{\ddagger}$ & 46 & 2010 & \\
\hline Allergy tablets/antihistamines ${ }^{\ddagger}$ & 70 & 2010 & \\
\hline Analgesics, price per day ${ }^{\ddagger}$ & 4.24 & 2010 & \\
\hline \multicolumn{4}{|c|}{$\begin{array}{l}\text { *Mean of prices from Southern Health Care Region, Northern Health Care Region } \\
\text { and Stockholm County Council. }\end{array}$} \\
\hline \multicolumn{4}{|c|}{${ }^{\dagger}$ Mean of prices from Skåne and Stockholm regions. } \\
\hline \multicolumn{4}{|l|}{${ }^{\ddagger}$ Mean of different brands. } \\
\hline \multicolumn{4}{|c|}{$E R=e m e r g e n c y$ room, MFNS=mometasone furoate nasal spray. } \\
\hline
\end{tabular}

and between HRQoL and MSS was assessed by regression analysis. Costs and HRQoL were then entered into the model per day and MSS level (see scatter graphs of costs or HRQoL and MSS in Figures 1 and 2). The slope and intercept for healthcare costs and for indirect costs were estimated using an ordinary least square (OLS) regression, while a Tobit regression was used for the costs of drugs and transportation. The indirect cost estimate was adjusted for the share of students/unemployed in the sample. The Tobit model was used when the dependent variable had a large proportion of zero values since the Tobit regression is suitable in the case of a censoreddependent variable. OLS was chosen for the regression on HRQoL by MSS. The OLS and Tobit methods yielded very similar results in all cases both for costs and HRQoL. Correlation between MSS, EQ-5D, EQ-VAS, and MSSUI was tested with the Spearman rank correlation test. P-values are two-sided with the significance level set at 0.05 . Data management and statistical analyses were performed using STATA 11 (StataCorp, 2009, College Station, Texas, USA).

\section{Sensitivity analyses}

In the sensitivity analyses the base case acts as a reference point and features of the base case are changed one at a time. Additional sensitivity analyses run base case with the cost of one pack of amoxicillin instead of two packs. The two final analyses conservatively assume no relationship between costs and MSS and hence only include the cost of the intervention pharmaceuticals.

\section{Results}

The base case results are presented in Table 2. MFNS 200 $\mathrm{gg}$ twice daily for 15 days was compared with amoxicillin 500mg three times daily for 10 days or non-active treatment using EQ-5D utilities. MFNS $200 \mu \mathrm{g}$ twice daily was the dominant treatment - that is, costs were reduced and QALYs increased in both comparisons. However, none of the regressions estimated for costs and generic HRQoL showed a strong relationship with MSS.

Results from the base case and sensitivity analyses are shown in Table 3. MFNS $200 \mu \mathrm{g}$ twice daily compared favourably with amoxicillin or non-active treatment in the sensitivity analysis regardless of HRQoL measurement used. Only counting the cost of intervention, the incremental cost-effectiveness ratio was SEK 273,150 compared with non-active treatment.

Table 4 shows the rank correlation between EQ-5D, EQ-VAS, MSSUI, and the disease severity measurement MSS. There is a high correlation between MSS and MSSUI, which is expected since MSSUI

Table 2. Base case model output (SEK, 2010)

\begin{tabular}{|c|c|c|c|c|c|}
\hline & MFNS $200 \mu \mathrm{g}$ twice daily & \multicolumn{2}{|c|}{ Amoxicillin 500mg } & \multicolumn{2}{|c|}{ Non-active treatment } \\
\hline \multicolumn{6}{|l|}{ Direct costs } \\
\hline Intervention drug costs & 166 & 197 & -31 & 0 & 166 \\
\hline Other drug costs & 17 & 20 & -4 & 21 & -4 \\
\hline Total costs & 7,568 & 7,817 & -249 & 7,667 & -99 \\
\hline QALYS & 0.0300 & 0.0293 & 0.0005 & 0.0326 & 0.0006 \\
\hline ICER & & & Cost-saving & & Cost-saving \\
\hline
\end{tabular}




\section{Figure 1. Scatter graph of cost input by Major Symptom Score (MSS)}
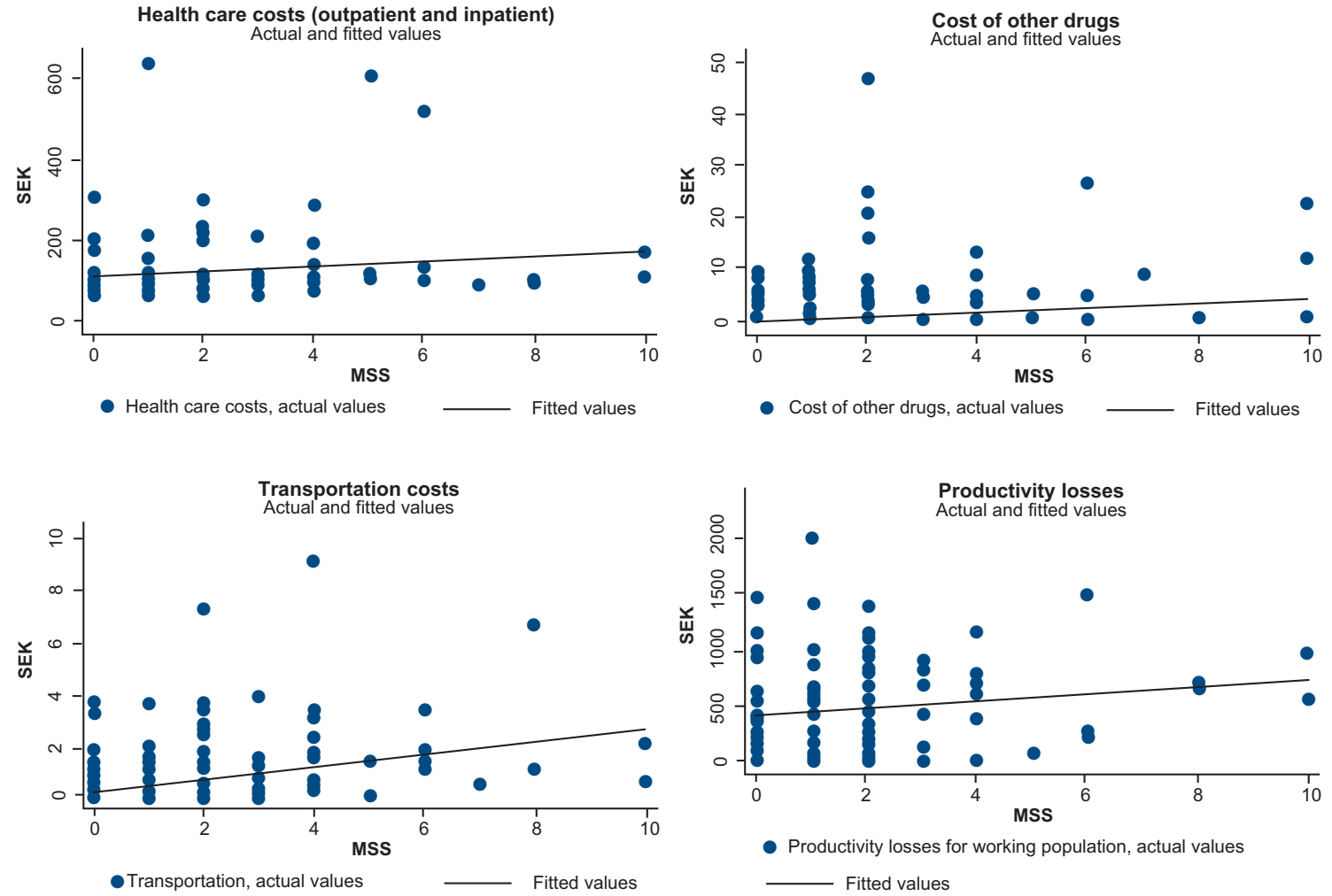

Figure 2. Scatter graph of utility weights by Major Symptom Score (MSS)
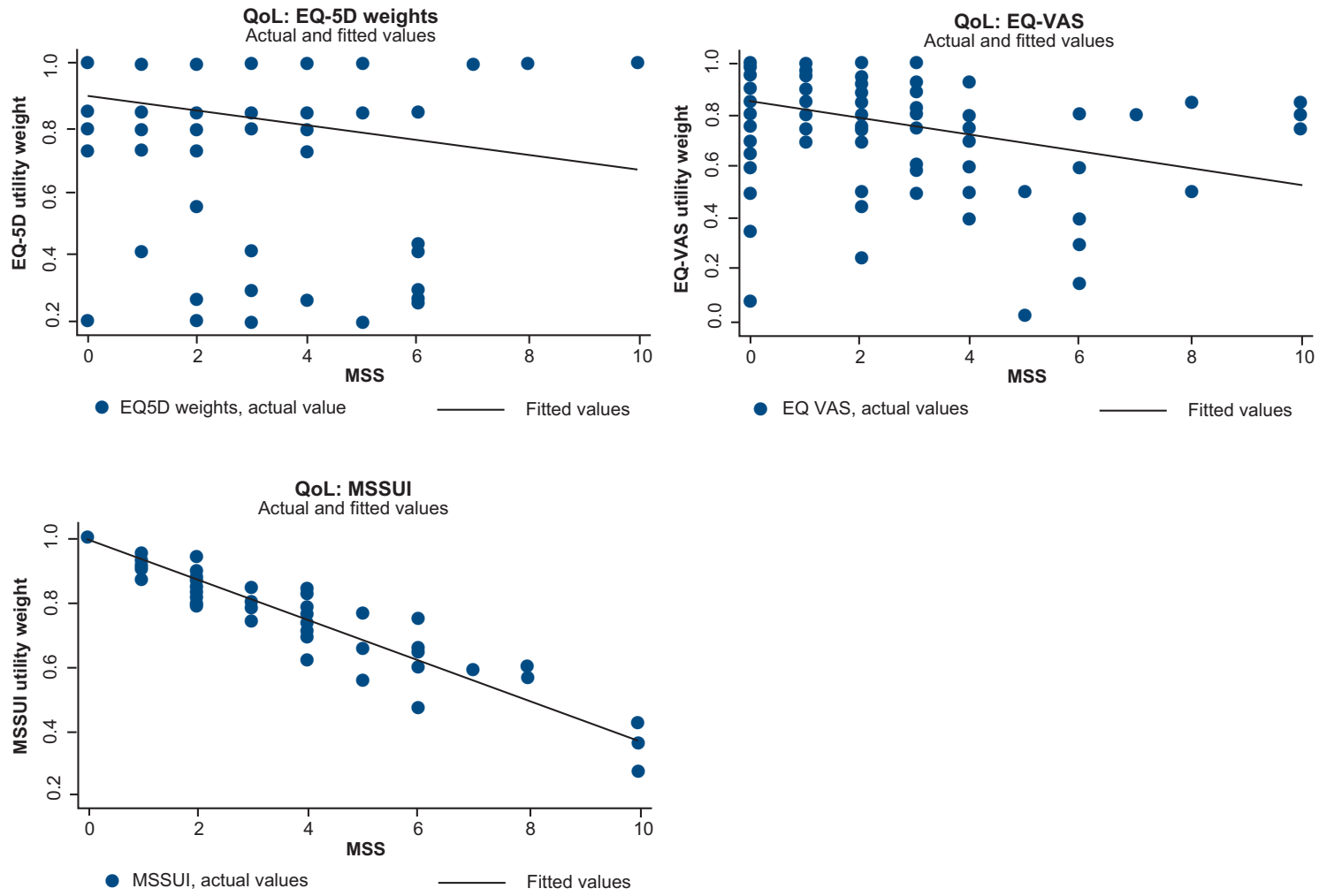


\begin{tabular}{|c|c|c|c|c|c|c|c|}
\hline \multicolumn{3}{|r|}{ Comparison } & Utility measure & Other & $\Delta$ Costs & $\triangle \mathrm{QALYs}$ & ICER \\
\hline \multirow{2}{*}{\multicolumn{2}{|c|}{$\begin{array}{l}\text { Base } \\
\text { case }\end{array}$}} & MFNS $200 \mu \mathrm{g}$ twice daily/amoxicillin 500mg x 3 & EQ-5D & - & -249 & 0.00050 & - \\
\hline & & MFNS $200 \mu \mathrm{g}$ twice daily/non-active treatment & EQ-5D & - & -99 & 0.00061 & - \\
\hline \multirow{7}{*}{ 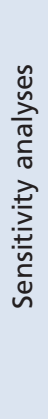 } & \multirow{2}{*}{1} & MFNS $200 \mu \mathrm{g}$ twice daily/amoxicillin 500mg x 3 & EQ-VAS & - & -249 & 0.00070 & - \\
\hline & & MFNS $200 \mu \mathrm{g}$ twice daily/non-active treatment & EQ-VAS & - & -99 & 0.00085 & - \\
\hline & \multirow[t]{2}{*}{2} & MFNS $200 \mu \mathrm{g}$ twice daily/amoxicillin 500mg x 3 & MSSUI & - & -249 & 0.00130 & - \\
\hline & & MFNS $200 \mu \mathrm{g}$ twice daily/non-active treatment & MSSUI & - & -99 & 0.00158 & \\
\hline & 3 & MFNS $200 \mu \mathrm{g}$ twice daily/amoxicillin 500mg x 3 & $E Q-5 D$ & $\begin{array}{l}\text { Amoxicillin price } \\
\text { of } 1 \text { pack }\end{array}$ & -150 & 0.00050 & - \\
\hline & \multirow[t]{2}{*}{4} & MFNS $200 \mu \mathrm{g}$ twice daily/amoxicillin 500mg x 3 & EQ-5D & $\begin{array}{l}\text { Only intervention } \\
\text { costs included }\end{array}$ & -31 & 0.00050 & - \\
\hline & & MFNS $200 \mu \mathrm{g}$ twice daily/non-active treatment & EQ-5D & $\begin{array}{l}\text { Only intervention } \\
\text { costs included }\end{array}$ & 166 & 0.00061 & 273,150 \\
\hline
\end{tabular}

ICER=incremental cost-effectiveness ratio, MFNS=mometasone furoate nasal spray, MSSUI=Major Symptom Score Utility Index, QALY=quality-adjusted life years

Table 4. Spearman correlation between utility measurements EQ-VAS, EQ-5D, MSSUI and disease severity measurement MSS ( $p$ values in parentheses)

\begin{tabular}{lllll} 
& EQ-VAS & EQ-5D & MSSUI & MSS \\
\hline EQ-VAS & 1 & & & \\
\hline EQ-5D & 0.4561 & 1 & & \\
& $(0.0000)$ & & & \\
\hline \multirow{2}{*}{ MSSUI } & 0.378 & 0.2428 & 1 & \\
& $(0.0000)$ & $(0.0035)$ & & 1 \\
\hline MSS & -0.3934 & -0.2296 & -0.9695 & \\
& $(0.0000)$ & $(0.0058)$ & $(0.0000)$ & \\
\hline
\end{tabular}

MSS=Major Symptom Score, MSSUI=Major Symptom Score Utility Index.

is derived from the same instrument that measures the MSS sum of scores. EQ-VAS was better correlated with MSS than EQ-5D with MSS. All correlation coefficients are significantly different from 0 .

\section{Discussion}

\section{Main findings}

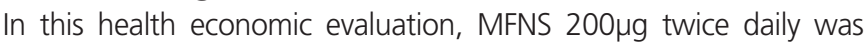
found to be the dominant or cost-saving therapy in acute rhinosinusitis compared with amoxicillin or non-active treatment as it both reduced costs and increased QALYS. There is no explicit cost per QALY threshold in Sweden, but average accepted cost per QALY in TLV (Dental and Pharmaceutical Benefits Agency) decisions was 36,000 Euros in 2008. ${ }^{14}$

\section{Strengths and limitations of this study}

The regression method chosen was considered appropriate with regard to the kind of dependent variable, core regression model assumptions, and applicability in predicting point estimates for the acute rhinosinusitis model. The low level of significance in the relationship between MSS and costs could partly depend on the fact that the cost variable covers a 15-day period during which MSS potentially changed, i.e. the MSS measurement on day 15 only reflects the disease severity that day. A patient with moderate disease, perhaps necessitating absence from work, could at day 15 arrive at the same MSS as a patient who experienced mild disease. For a disease that often resolves without treatment, we would also expect to find a variation in the resources spent to treat it. Some patients got better as a result of a costly treatment (pharmaceuticals and healthcare consumption) while some patients got better despite spending very little on treatment.

Three HRQoL measurements were used in the analysis. EQ-5D has the advantage of being widely used and accepted by reimbursement bodies such as TLV (Dental and Pharmaceutical Benefits Agency) in Sweden. The five dimensions concerning mobility, self-care, usual activities, pain, and anxiety have been proved a valid generic HRQoL measurement for general health status, but its validity as an outcome measure in rhinosinusitis has to our knowledge not previously been tested. ${ }^{15}$ The HRQoL (EQ-5D and EQ-VAS) at day 15 in the observational study is in line with results for the general population in Sweden and France. 16,17 The EQ-VAS scale concerns general health and is therefore sensitive to health issues unrelated to acute rhinosinusitis. On the other hand, the absence of questions directing what the patient should take into account when assessing their health status makes the EQ-VAS more sensitive to changes in acute rhinosinusitis than the EQ-5D. The low correlation between EQ-5D and MSS probably depends on the fact that the pain/discomfort is the only dimension measured by both instruments. The correlation between EQ-VAS and MSS was somewhat higher but still rather low. MSSUI is a relevant outcome measure for the treatment of acute rhinosinusitis but is of limited value in a health economic model. The gains in HRQoL measured by disease-specific measurements cannot be compared with gains in HRQoL for other diseases; instead of QALYs it produces acute rhinosinusitis-adjusted life years. Generic instruments are often not sensitive enough to detect changes in less severe diseases such as rhinosinusitis. This problem has been discussed by Jenkinson and coworkers. ${ }^{18}$ Other authors have focused on the lack of valid responsive outcome measures for patients with acute sinusitis. ${ }^{15}$ 
Bachert and Meltzer demonstrated significant improvement in disease-specific quality of life among effectively treated patients with moderate acute rhinosinusitis. ${ }^{19}$ However, the SNOT-20 questionnaire used in that study was not originally developed for acute rhinosinusitis.

The model has a number of important strengths. The combination of the controlled randomised study results of treatment outcome and the observational study which considers everyday clinical practice means that disease progression and costs are credible. The similarities in design of the randomised clinical study and the observational study are a further advantage for the costeffectiveness analysis. A randomised clinical trial is often directed by a rigid protocol stipulating conditions that are rarely met in actual clinical practice. The fact that the observational study shows baseline patient characteristics very similar to those of the clinical study ${ }^{4}$ supports the conclusion that the clinical study data reliably describe a disease progression relevant for actual practice and hence for health economic modelling. The access to two generic utility measurements (EQ-5D and EQ-VAS) and one disease-specific (MSSUI) makes the results more robust although none of them are optimal. Difficulties to find a generic instrument that is sensitive to changes of less severe diseases of short duration have been reported by others. ${ }^{20}$

A limitation of the cost-effectiveness model is that the often insignificant connection between costs and MSS, and HRQoL and MSS, bring an uncertainty to the results for which the model does not account. The costs by MSS and HRQoL by MSS functions are the same for each treatment arm, however, and therefore the uncertainty does not necessarily favour any treatment arm. If we conservatively assume that there is no relationship between MSS and costs, the incremental cost-effectiveness ratio reaches SEK 273,150 when MFNS $200 \mu \mathrm{g}$ twice daily is compared with non-active treatment. Another limitation is the fact that there is only one published randomised controlled clinical study with MFNS as monotherapy in acute rhinosinusitis ${ }^{4}$ from which the effect size estimates were taken.

Implications for future research, policy and practice Antibiotics are of little or no benefit in most cases of routine treatment of uncomplicated acute rhinosinusitis because of the risk of increased antibiotic resistance, unnecessary side-effects, and higher cost of medical care. ${ }^{5,21}$ Acute rhinosinusitis has been shown to be associated with substantial costs to the healthcare system, the individual, and to society as a whole. ${ }^{6}$ A change in treatment practice with a reduced prescription of antibiotics could save money and improve HRQoL for patients diagnosed with acute rhinosinusitis.

In view of increasing antibiotic resistance in society, the present study shows promising results. Meltzer and co-workers ${ }^{4}$ showed that rhinosinusitis can be treated successfully without antibiotics, and we have shown that costs can be saved at a societal level in doing so.

\section{Conclusions}

Using observational and clinical data to model acute rhinosinusitis in the Swedish setting shows that treatment with MFNS 200 $\mu$ g twice daily results in lower costs and increased QALYs compared with the alternatives (amoxicillin or non-active treatment). The quality of life instruments EQ-5D and EQ-VAS probably have limited sensitivity for detecting differences in HRQoL in patients with acute mild to moderate rhinosinusitis. In spite of that, the model simulation shows that treatment with MFNS in most analyses results in lower costs than amoxicillin or non-active treatment and thus is cost-saving. Consequently, MFNS 200 $\mu$ g twice daily provides an attractive alternative to antibiotics in the treatment of acute rhinosinusitis.

Handling editor Tjard Schermer
Statistical review Gopal Netuveli

Acknowledgements The authors thank Patrick Svarvar, Merck, for expertise input.

Conflicts of interest $P O$ is employed by Boehringer-Ingelheim $A B$. $J L$ is employed by and owns stock and stock options in Merck \& CO. PS has received funding from MSD, GSK and Novartis and has received honoraria for studies and advisory boards. JS and GRT declare that they have no conflicts of interest in relation to this article.

Contributorship JS: conception and design, interpretation, writing manuscript, final approval of manuscript. JL: interpretation, revising manuscript, final approval of manuscript. PO: conception and design, interpretation, revising manuscript, final approval of manuscript. PS: conception and design, interpretation, revising manuscript, final approval of manuscript. GRT: conception and design, interpretation, writing manuscript, final approval of manuscript.

Funding This study was financed by MSD, Sollentuna, Sweden.

\section{References}

1. Fokkens WJ, Lund VJ, Mullol J. European Position paper on rhinosinusitis and nasal polyps 2007. Rhinology 2007;45(Suppl 20):1-139.

2. Bhattacharyya N. Contemporary assessment of the disease burden of sinusitis. Am J Rhinol Allergy 2009;23(4):392-5. http://dx.doi.org/10.2500/ajra.2009.23.3355

3. Andre $M$, Vernby $A$, Odenholt I, et al. [General practitioners prescribed less antibiotics but used the CRP test more. Diagnosis-prescription studies in 2000-2005]. Lakartidningen 2008;105(41):2851-4.

4. Meltzer EO, Bachert C, Staudinger H. Treating acute rhinosinusitis: Comparing efficacy and safety of mometasone furoate nasal spray, amoxicillin, and placebo. J Allergy Clin Immunol 2005;116:1289-95.

http://dx.doi.org/10.1016/j.jaci.2005.08.044

5. Young J, De Sutter A, Merenstein D, et al. Antibiotics for adults with clinically diagnosed acute rhinosinusitis: a meta-analysis of individual patient data. Lancet 2008;371(9616):908-14. http://dx.doi.org/10.1016/S0140-6736(08)60416-X

6. Wasserfallen J-B, Livio F, Zanetti G. Acute rhinosinusitis. A pharmacoeconomic review of antibacterial use. Pharmacoeconomics 2004;22(13):829-37.

http://dx.doi.org/10.2165/00019053-200422130-00002

7. Revicki DA, Margolis MK, Thompson CL, Meltzer EO, Sandor DW, Shaw JW. Major symptom score utility index for patients with acute rhinosinusitis. Am J Rhinol Allergy 2011;25(3):99-106. http://dx.doi.org/10.2500/ajra.2011.25.3575

8. Stjärne P, Odebäck P, Ställberg B, Lundberg J, Olsson P. High costs and burden of illness in acute rhinosinusitis: real life treatment patterns and outcomes in Swedish primary care. Prim Care Respir J 2012;21(2):174-9. http://dx.doi.org/10.4104/pcrj.2012.00011

9. Läkemedelsverket (Medical Products Agency). [Pharmaceutical treatment of rhinosinusitis - treatment guidelines 2005]. In Swedish.

10. Swedish Association of the Pharmaceutical Industry (LIF). FASS (Pharmaceutical Specialties in Sweden). Stockholm, 2010.

11. Statistics Sweden. Mean annual income 2008. 2010. Available from: http://www.scb.se/Pages/TableAndChart__28871.aspx.

12. Dolan P. Modeling valuations for EuroQol health states. Med Care 1997;35(11):1095-108. http://dx.doi.org/10.1097/00005650-199711000-00002

13. Dolan P, Gudex C, Kind P, Williams A. A social tariff for EuroQol: results from a UK general population survey. 1995. Available from: http://www.york.ac.uk/media/che/documents/papers/discussionpapers/CHE\%20Disc ussion\%20Paper\%20138.pdf.

14. Hugosson K, Engstrom A. Threshold value for a QALY - correlation with disease severity and decision uncertainty. Value Health 2008;11(3):A49. 
http://dx.doi.org/10.1016/S1098-3015(10)70165-0

15. Linder J, Singer $D$, van den Ancker $M$, Atlas $S$. Measures of health-related quality of life for adults with acute sinusitis: a systematic review. I Gen Intern Med 2003;18:390-401. http://dx.doi.org/10.1046/j.1525-1497.2003.20744.x

16. Burström $K$, Johannesson $M$, Diderichsen $F$. Swedish population health-related quality of life results using the EQ-5D. Qual Life Res 2001;10:621-35. http://dx.doi.org/10.1023/A:1013171831202

17. Perneger $T$, Combescure $C$, Courvoisier D. General population reference values for the French version of the EuroQol EQ-5D health utility instrument. Value Health 2010;13(5):631-5. http://dx.doi.org/10.1111/.1524-4733.2010.00727.x

18. Jenkinson C, Gray A, Doll H, Lawrence K, Keoghane S, Layte R. Evaluation of index and profile measures of health status in a randomized controlled trial. Med Care 1997;35(11):1109-18. http://dx.doi.org/10.1097/00005650-199711000-00003

19. Bachert C, Meltzer EO. Effect of mometasone furoate nasal spray on quality of life of patients with acute rhinosinusitis. Rhinology 2007;45(3):190-6.

20. Brazier JE, Walters SJ, Nicholl JP, Kohler B. Using the SF- 36 and Euroqol on an elderly population. Qual Life Res 1996;5:195-204. http://dx.doi.org/10.1007/BF00434741

21. Ryan D. Management of acute rhinosinusitis in primary care: changing paradigms and the emerging role of intranasal corticosteroids. Prim Care Respir 1 2008;17(3):148-55. http://dx.doi.org/10.3132/pcrj.2008.00050

22. Stockholm County Council. Price List. 2010. Available from: http://extern.bkv.sll.se/samv.sthlm-gotland/Prislistor/prislist_utomlvard.htm.

23. Southern Health Care Region. Price List. 2010. Available from: http://www.skane.se/templates/Page.aspx?id=190940.

24. Northern Health Care Region. Price List. 2010. Available from: http://www.norrlandstingen.se/lankar_prislistor.htm.

25. SL. 2010. Available from: http://sl.se/sv/ResenarNalja-biljettNara-biljetter/.

26. Skånetrafiken. 2010. Available from: http://www.skanetrafiken.se/templates/ InformationPage.aspx?id=26872\&epslanguage $=$ SV.

27. Läkemedelsindustriföreningen (LIF). FASS (Pharmaceutical Specialties in Sweden). Stockholm, 2010

Available online at http://www.thepcrj.org 